\title{
Knowledge of vitreomacular traction (VMT) scenarios: Is doing nothing still a beneficial alternative and, if so, when?
}

\author{
Focke Ziemssen $^{1} \cdot$ Karl Ulrich Bartz-Schmidt ${ }^{1} \cdot$ Spyros Dimopoulos $^{1}$
}

Received: 3 December 2015 / Accepted: 17 December 2015 /Published online: 18 February 2016

(C) Springer-Verlag Berlin Heidelberg 2016

Keywords Vitreomacular adhesion · Ocriplasmin .

Vitrectomy $\cdot$ SD-OCT $\cdot$ Natural history

It is to the great merit of the International Vitreomacular Traction (VMT) Study Group that the new classification brought clarity by reflecting the standards of modern ocular coherence (OCT) imaging [1]. These activities go back to the pioneers of pharmacologic vitreolysis [2]. The dream of replacing sophisticated intraocular surgery with a single administration of a vitreolytic agent is intriguing. The illusion of a simple injection as the solution to most cases might have slightly blurred the borders, when the medical art of doing nothing still might be the best choice anyway. A more realistic assessment started after the sobering news of transient or even permanent toxicity related to ocriplasmin [3].

It is thanks to Peter Stalmans that the body of evidence regarding the natural history of VMT has increased further [4]. The retrospective study was funded, but not influenced by ThromboGenics. The excellent flowchart provided in the study (Figure 4: outcomes by baseline disease) displays how the different subgroups developed during the short follow-up period of 11 months:

Focke Ziemssen

focke.ziemssen@med.uni-tuebingen.de

1 Centre for Ophthalmology, Eberhard-Karls-University, Schleichstr. 12, 72076 Tuebingen, Germany
- Patients with vitreomacular adhesion (VMA) showed a similar rate of progression and resolution. However, the large majority $(95.2 \%)$ did not receive any treatment, though the amount of (on request) reported metarmophopsia was found to be around one in five (18\%).

- In patients with vitreomacular traction (VMT) the chance of spontaneous resolution $(22.7 \%)$ and the risk of undergoing vitrectomy (25.6\%) appeared to be balanced [4]. In particular regarding the latter group, bias cannot be ruled out, as the cohort of a tertiary centre might not be representative in terms of comorbidities. Moreover, the decision might strongly depend also on the more or less aggressive approach and information policy of the individual surgeon.

- The number of cases with macular holes and concomitant VMT was quite small (9.5\%). The rate of surgery did not differ from other cases with macular holes $(88.7 \%$ vs. $86.4 \%$ ). It is a limitation of the study that the author did not apply the differentiation of his own classification (small vs. large holes) and the individual peculiarity that silicone oil was used during every surgery. Therefore, we do not wish to comment on the decision regarding vitrectomy for macular holes, which might be recommended often, - at least in our experience, except in cases with a poor prognosis due to the long duration (not so seldom) and/or any end-stage atrophy.

This study is of importance, as the MIVI trials did not provide comprehensive knowledge of natural disease history in the absence of a real "placebo" group [2]. In addition, the follow-up of the RCTs was quite short considering the situation that the story, including partial resolution, is not over after 4 weeks or 6 months, and the rate of spontaneous VMT resolution has been found to continuously rise over time [5]. 
Watchful waiting is also associated with the risk of a gradual loss of vision and the formation of macular holes. Thus, what are the three key messages regarding VMT treatment at the beginning of 2016 ?

1. It is crucial to define the best candidates with a more favourable response to ocriplasmin: traction with a focal vitreomacular adhesion $(<1500 \mu \mathrm{m})$, the absence of any epiretinal membrane (ERM), in cases of macular holes showing traction limited to a smaller area $(\leq 250 \mu \mathrm{m})$, the presence of subretinal fluid, and younger age $[6,7]$. Incidentally, most of these factors also seem to increase the spontaneous resolution rate $[5,6,8]$.

2. It is not easy to select the right patients. Even within prospective trials, one in five retina specialists failed to select the appropriate phenotype [7]. Facing a relevant learning curve, dual control and in-process monitoring can be used to evaluate the individual failure rate of treating the wrong subjects. This selection more closely resembles carefully cherry picking, as the majority of VMT patients (50-60\%) do have an epiretinal membrane [6]. An OCT scan crossing the disc helps in evaluating the stage of vitreous detachment in detail. The presence of symptoms does not necessarily meet the requirements of being bothered or disturbed.

3. In the absence of dramatic changes during a short-term follow-up period, there is time to prepare and engage in shared-decision making. The best practice requires an extensive amount of information, but each intervention is elective rather than urgent [6]. Although gradual loss of vision might occur with delayed treatment, there is no need to put patients under time pressure. Even in cases with a history of recent vision loss, there will be time to postpone the final decision to a second appointment.

4. There is still no justification for a general recommendation, e.g. in favour of pharmacologic vitreolysis [6]. Candidates have to be informed about the nonetheless high rate of surgery subsequent to ocriplasmin treatment. Even in good candidates, ocriplasmin remains a two-step approach for a large proportion. This means that it is more likely that there is no resolution, and consequently vitrectomy will have to be scheduled. Partial resolution represents scenarios when good advice becomes difficult. It has to be considered whether a trial should evaluate the outcome of the randomisation of ocriplasmin treatment against the more cost-efficient primary vitrectomy, if there is a wish for therapy [9].

The discussion of this complex situation should include many influential factors. Time and experience are required to assess the severity of symptoms, in particular the impact on daily living. Additional risk factors of vitrectomy, the duration of pre-existing VMT, and the personality of the affected person have to be taken into account. Adverse events related to ocriplasmin seem to be rare [3] and transient problems need about 6 months to resolve. In the case of changes in the outer retina, persistence is uncommon (10\%). Despite these concerns, the future will be brighter. Swept source images will further resolve uncertainties. Based on a better understanding of the resolution process, it will be easier to give more thorough and sound advice to the individual patient.

\section{Compliance with ethical standards}

Potential conflict of interest Focke Ziemssen discloses a potential conflict of interest due to consulting fees from Alimera, Allergan, Bayer HealthCare, Novartis and speaker fees from Alcon, Alimera, Allergan, Bayer HealthCare, Heidelberg Engineering, Novartis.

Spyros Dimopoulos discloses a potential conflict of interest due to travel expenses covered by Alcon.

Karl-Ulrich Bartz-Schmidt discloses a potential conflict of interest due to consulting for Alcon and grants as well as speaker fees from Acucela, Alcon, Allergan, Boehringer, Genentech Heidelberg Engineering, Novartis and Roche.

\section{References}

1. Duker JS, Kaiser PK, Binder S et al (2013) The international vitreomacular traction study group classification of vitreomacular adhesion, traction, and macular hole. Ophthalmology 120:26112619

2. Stalmans P, Benz MS, Gandorfer A et al (2012) Enzymatic vitreolysis with ocriplasmin for vitreomacular traction and macular holes. N Engl J Med 367:606-615

3. Small KW, Shaya FS, La Fontaine M (2015) Post-market experience with ocriplasmin including chronic electrophysiologic changes. Ophthalmic Surg Lasers Imaging Retina 46(9):956-962

4. Stalmans P (2015) A Retrospective Cohort Study in Patients With Tractional Diseases of the Vitreomacular Interface (ReCoVit). Graefes Arch. Clin. Exp. Ophthalmol. E-Pub

5. Dimopoulos S, Bartz-Schmidt KU, Gelisken F, Januschowski K, Ziemssen F (2015) Rate and timing of spontaneous resolution in a vitreomacular traction group: should the role of watchful waiting be re-evaluated as an alternative to ocriplasmin therapy? $\mathrm{Br} \mathrm{J}$ Ophthalmol 99(3):350-353

6. Folk JC, Adelman RA, Flaxel CJ, Hyman L, Pulido JS, Olsen TW (2015) Idiopathic Epiretinal Membrane and Vitreomacular Traction Preferred Practice Pattern ${ }^{\circledR}$ Guidelines. Ophthalmology

7. Kaiser P. The OASIS Study: Evaluating Ocriplasmin for the Treatment of Symptomatic VMA/VMT Including Macular Hole Over 2 Years. ThromboGenics press release. 18 Nov 2015 http:// www.thrombogenics.com/content/thrombogenics-reports-positive2-year-results-oasis-study-jetrea $\% \mathrm{C} 2 \% \mathrm{AE}$-ocriplasmin-treatment

8. Chatziralli I, Theodossiadis G, Parikakis E, Datseris I, Theodossiadis P (2015) Real-life experience after intravitreal ocriplasmin for vitreomacular traction and macular hole: a spectral-domain optical coherence tomography prospective study. Graefes Arch. Clin. Exp. Ophthalmol. E-Pub

9. Chang JS, Smiddy WE (2014) Cost evaluation of surgical and pharmaceutical options in treatment for vitreomacular adhesions and macular holes. Ophthalmology 121(9):1720-1726 